\title{
Splenomegaly as a Primary Manifestation of Gaucher Disease in a Young Adult Woman
}

\author{
Giuseppe Merra ${ }^{a}$ Antonio Dal Lago ${ }^{a}$ Roberta Riccib \\ Daniela Antuzzi ${ }^{\text {b }}$ Giovanni Gasbarrini ${ }^{a}$ \\ Antonio Gasbarrini ${ }^{a}$ Giovanni Ghirlanda ${ }^{a}$
}

Departments of a Internal Medicine and ${ }^{b}$ Pediatrics, Catholic University of Sacred

Heart, Rome, Italy

\section{Key Words}

Gaucher disease - Lysosomal storage disease · Splenomegaly · Accumulation . Macrophages

\begin{abstract}
Gaucher disease is the most common lysosomal storage disease. It is caused by the defective activity of acid $\beta$-glucosidase, which results in the accumulation of lipid glucocerebroside in macrophages throughout the body. In this case report we describe the case of a young adult woman with splenomegaly as the primary manifestation of this pathology. This is a case of type 1 Gaucher disease because there is a lack of primary neurological involvement but we have, instead, an age-independent involvement of the visceral organs. It is very important to classify or characterize these patients in a precise manner and to make a complete diagnosis with the help of the many diagnostic resources now at our disposal, especially with genetics, radiology and new techniques of advanced microscopy, also because Gaucher disease requires a long and complex management from early life to adulthood.
\end{abstract}

\section{Introduction}

Gaucher disease is the most common lysosomal storage disease [1,2]. It is caused by the defective activity of acid $\beta$-glucosidase, which results in the accumulation of lipid glucocerebroside in macrophages throughout the body $[3,4]$. There are many manifestations of Gaucher disease, such as hepatosplenomegaly, anemia, thrombocytopenia and bone marrow infiltration with characteristic storage cells, Gaucher 
cells and bony lesions [5]. Three forms of Gaucher disease have been identified [6]. The most common form is type 1 , characterized by a lack of primary neurological involvement but with involvement of the visceral organs to varying degrees. However, neurological involvement occurs early during disease progression in type 2 disease and later in type 3 disease. In fact types 2 and 3 have been termed acute and subacute neuronopathic, respectively, based on the rapidity of progression of central nervous system deterioration and at onset [7].

\section{Case Report}

The patient, an 18-year-old woman, came under our observation due to the persistence, for more than six months, of widespread articular pain, especially during the night, easy fatigue and the occasional occurrence of thrombocytopenia. For these reasons she had already been to another hospital and a not better specified diagnosis of 'autoimmune-based illness' had been formulated, for which cyclosporine therapy was prescribed, pending a probable splenectomy. During the stay in our hospital the patient complained of bone pain, particularly articular and in the lower limbs, although to a lesser degree than before beginning the previously mentioned immunosuppressive therapy, and easy fatigue. No signs worth noting emerged from anamnesis. On objective examination the patient was found to be in a reasonably good general condition, alert and well oriented in time and space, with normal facies, negligible decubitus and no signs of bilateral peripheral edemas. The skin had a normal blood supply and was normally hydrated, and the subcutaneous panniculus adiposus was normally represented and distributed. The muscular mass was normotonic and normotrophic and the superficial lymph node system was undamaged. Locoregional objective examination was negative except for the presence of impressive splenomegaly.

Specialist neurological objective examination was completely negative. The vital parameters monitored were normal. The results of the hematochemical examinations carried out were normal except for a slight enzymatic cholestasis $(\gamma-\mathrm{Gt}<2 \times)$ and a slight hypertriglyceridemia. Protein electrophoresis showed an insignificant increase in alpha-2 globulin and a slight reduction in beta-2 globulin. Blood count showed hemoglobin equal to $10 \mathrm{~g} / \mathrm{dl}$, hematocrit equal to $29.4 \%$, a red blood cell count of 3,720,000, a white blood cell count of 5,270, MCV equal to $79.1 \mathrm{fl}$ and a platelet count of 115,000. Blood coagulation tests showed a PT of $68.4 \%$ and an APTT of $37.80 \mathrm{~s}$. Abdominal nuclear magnetic resonance imaging (NMRI) showed: 'a considerably enlarged liver (cranio-caudal diameter $24 \mathrm{~cm}$ ); splenomegaly (cranio-caudal diameter $22 \mathrm{~cm}$, transverse diameter $11 \mathrm{~cm}$ ) with dilation of the spleen vein'. For a more in-depth diagnostic analysis the patient was subjected to a hepatic biopsy with the diagnosis of 'hepatic tissue characterized by marked hypertrophy of Kupffer cells (PGM-1 positive to immunohistochemistry) with extensive cytoplasm with a wrinkled appearance, eosinophilic with PAS diastase staining: morphological report indicative of Gaucher disease'. Based on these results we carried out an analysis of the glucocerebrosidase gene through amplification of a DNA sequence (PCR), detecting the presence of the N370S mutation in both alleles. The genetic test was then carried out on the rest of the family. A skeletal X-ray of the patient showed that the overall picture was within the norm except for the presence of structural alterations in the proximal metaphyseal and diaphyseal region of the right tibia, compatible with Gaucher disease', and a DEXA study showed 'particularly low BMD compared to normal limits for the same gender and age'. NMRI of the cranium was negative. On completion of the diagnostic procedure, as a result, the patient was discharged with enzymatic replacement therapy.

The follow-up of this patient after a year of therapy indicates that her present condition is good, she has neither cholestasis nor hypertriglyceridemia and there is satisfactory reduction in splenomegaly, hypersplenism and articular pain. However we are planning to follow the patient for a long time.

\section{Discussion}

Certainly, apart from the general (fatigue, bone pain, etc.) or laboratory (anemia, thrombocytopenia, etc.) signs and suspected pathology detected by NMRI, as in this case, the diagnosis of Gaucher disease is established histologically by examining bone marrow or tissue from liver or spleen biopsies [8]. However histological diagnosis of Gaucher 
disease is not always reliable because it is not easy to differentiate the abnormal macrophages, in fact other similar cells are present in hemorrhagic disorders, leukemia, lymphoma, osteomyelitis and Legg-Calvé-Perthes disease, in fact, finally the histological diagnosis of Gaucher requires an invasive procedure such as a bone marrow biopsy and only occasionally and unfortunately a liver or spleen biopsy is enough for diagnosis.

An alternative approach used by us is that of DNA analysis [6]. Of more than 200 different mutations identified in Gaucher disease patients, only five have any general frequency [4]. The N370S and L444P mutations have some predictive value and these mutant alleles account for about half of identified Gaucher disease patients of European descent. The N370S mutation is the most common mutation in the Ashkenazi Jewish variant of type 1 Gaucher disease and is the most frequent mutation found in such patients in North America and Europe. The presence of a single N370S allele predicts non-neuronopathic disease [9], whereas N370S allele in conjunction with another mutant allele predicts more severe disease; the L444P homozygous state has a very high association with the neuronopathic variants such as type 2 or 3 Gaucher disease [10-12]. To classify or characterize patients with this pathology, a physical and neurological examination is necessary to exclude the presence of strabismus, saccadic initiation abnormalities of the eye, opisthotonic posturing and bulbar palsy/paresis [4].

This case is a case of type 1 Gaucher disease because there is a lack of primary neurological involvement but we have, instead, an age-independent involvement of the visceral organs. Symptomatic children designated as type 1 can experience variably progressive hepatosplenomegaly and/or hematologic abnormalities; in fact thrombocytopenia is often an early finding and anemia is somewhat less common and usually mild [6]. Growth retardation is a common and often unappreciated sign without concordance with progression of hepatosplenomegaly, but our patient showed regular growth [13]. Hepatomegaly can be severe and splenomegaly is present in most patients and may be marked [14], as in our case.

Osteopenia and osteoporosis have become increasingly diagnosed in recent years, especially in children, but osteoporosis by DEXA during puberty is very important. The skeletal involvement of Gaucher disease is characterized by the infiltration of bone marrow [15] with possible edema or ischemia, osteopenia with an abnormal decrease in mineral bone density and bone mass, localized or diffuse, Erlenmeyer flask deformity, osteonecrosis with bone death caused by chronic ischemic events and associated with acute pain, bone crisis that is a severe weakening episodic manifestation with a high fever, pathological fractures, general bone pain and asymptomatic growth retardation. The development of skeletal involvement in Gaucher disease is present in several ethnic groups as Ashkenazi Jewish, Dutch and Japanese.

For the treatment of Gaucher disease physicians were faced with the difficulty of treating patients before enzyme replacement therapy became available by ensuring the patients with high quality of life [6]. Splenectomy is a possible measure for correcting thrombocytopenia, but today it is generally deferred as long as possible because of the suspicion that it accelerates the deposition of glycolipid at other sites, but the evidence is anecdotal, and also because the response to therapy in this sense is good and splenectomy is carried out much less frequently, but if thrombocytopenia does not respond quickly to enzyme replacement therapy, splenectomy may be in order.

Recently the history of enzyme replacement therapy has been reviewed and there is no question that the therapy is effective, although it is extremely costly and the issue of 
dosage is often controversial. Other studies, especially with chemical inhibitors of glucosylceramide synthase, are still in progress.

\section{Conclusions}

Although Gaucher disease is a rare disorder, today many specialists, especially pediatricians [4], identify and will continue to identify patients with this pathology in their practice, so it is very important to classify or characterize these patients in a precise manner, make a complete diagnosis with the help of the many diagnostic resources now at our disposal, especially with genetics, radiology and new techniques of advanced microscopy, also because Gaucher disease requires a long and complex management from early life to adulthood. 


\section{References}

1 Butters TD: Gaucher disease. Curr Opin Chem Biol 2007;11:412-418.

2 Schmitz J, Poll LW, von Dahl S: Therapy of adult Gaucher disease. Haematologica 2007;92:148-152.

-3 Mignot C, Doummar D, Maire I, et al; French Type 2 Gaucher Disease Study Group: Type 2 Gaucher disease: 15 new cases and review of the literature. Brain Dev 2006;28:39-48.

-4 Grabowski GA: Recent clinical progress in Gaucher disease. Curr Opin Pediatr 2005;17:519-524.

5 Lutsky KF, Tejwani NC: Orthopaedic manifestations of Gaucher disease. Bull NYU Hosp Jt Dis 2007;65:37-42.

-6 Beutler E: Gaucher disease: multiple lessons from a single gene disorder. Acta Paediatr Suppl 2006;95:103-109.

7 Brady RO, Barton NW, Grabowski GA: The role of neurogenetics in Gaucher disease. Arch Neurol 1993;50:1212-1224.

$\checkmark 8$ Beutler E, Saven A: Misuse of marrow examination in the diagnosis of Gaucher disease. Blood 1990;76:646-648.

-9 Theophilus B, Latham T, Grabowski GA, Smith FI: Gaucher disease: molecular heterogeneity and phenotype-genotype correlations. Am J Hum Genet 1989;45:212-225.

10 Sidransky E: Gaucher disease: complexity in a 'simple' disorder. Mol Genet Metab 2004;83:6-15.

11 Tsuji S, Choudary PV, Martin BM, et al: A mutation in the human glucocerebrosidase gene in neuronopathic Gaucher's disease. N Engl J Med 1987;316:570-575.

-12 Tylki-Szymańska A, Millat G, Maire I, Czartoryska B: Types I and III Gaucher disease in Poland: incidence of the most common mutations and phenotypic manifestations. Eur J Hum Genet 1996;4:334-337.

13 Charrow J, Andersson HC, Kaplan P, et al: The Gaucher registry: demographics and disease characteristics of 1698 patients with Gaucher disease. Arch Intern Med 2000;160:2835-2843.

14 Grabowski GA, Andria G, Baldellou A, et al: Pediatric non-neuronopathic Gaucher disease: presentation, diagnosis and assessment. Consensus statements. Eur J Pediatr 2004;163:58-66.

15 Stowens DW, Teitelbaum SL, Kahn AJ, Barranger JA: Skeletal complications of Gaucher disease. Medicine (Baltimore) 1985;64:310-322. 